\title{
The Development and Degeneration of the Photoreceptor Outer Segment of the Fish Pineal Organ
}

\author{
Yuri OMURA and Mikio OGURI* \\ (Received March 25, 1971)
}

\begin{abstract}
The fine structures of fish pineal organ were investigated, with special reference to the photoreceptor cells during the developing and degenerating processes. Remarkable structural changes were detected in the outer segments of photoreceptor cells. From these changes in ultrastructures, the following eight types of outer segments were distinguished; 1) ciliary, 2) tubular, 3) partially lamellar, 4) wholly lamellar, 5) modifing lameilar, 6) distorting and swirling lamellar, 7) isolating lamellar, and 8) exhausted types.
\end{abstract}

It is well known that in general the outer segment of photoreceptor cell in vertebrate retina shows regularly arranged lamellar structure perpendicular to the longitudinal axis of the cell. However, the irregular alteration of ultrastructure detectable in the outer segment suggested the cyclic changes of development and degeneration of the photoreceptor cell. ${ }^{1-7)}$

As we have already reported, the outer segment of the photoreceptor cell in the fish pineal organ shows remarkable diversity under light and electron microscopies ${ }^{8-10}$

The present paper deals primarily with the development and degeneration of the outer segment of pineal photoreceptor cells in fish, to clarify whether the same ultrastructural changes are observable as described in retinal photoreceptor cell.

\section{Materials and Methods}

In this study we used the embryo, juvenile, adult and old specimens of Ayu, Plecoglossus altivelis, and the embryo and adult specimens of guppy, Poecillia reticulata.

The embryos of Ayu were obtained by the rearing of the eggs fertilized artificially and hatched in our laboratory in October, 1970. The juvenile fishes of Ayu were collected and fixed at Fisheries Laboratory of the University of Tokyo from January to March. The adult fishes of Ayu were obtained from the fish culture farm and the old fishes were supplied from the Aquarium of Fisheries Laboratory, the University of Tokyo. Adult guppies used in this study are the raised ones in our laboratory. For the examination of embryonic pineal organ, viviparous embryos were used.

Total head of fish was used for the fixation of pineal organ of small specimens, such as embryo and juvenile fish. In case of larger fish, only cutted pineal region was employed.

* Fisheries Laboratory, Faculty of Agriculture, Nagoya University, Nagoya, Japan. （大村百合.




These materials were fixed with $1 \% \mathrm{OsO}_{4}$ in MILLONIG's or SöRENSEN's phosphate buffer or in HAMA's collidin buffer.

After fixation for 1.5-2 hours, the specimens were dehydrated with an increasing ethanol series and embedded in epoxy resin. The ultra thin sections were cutted with PORTER-BLUME MT 1 ultra-microtome, stained with uranyl acetate and lead citrate solutions and observed with JEM T7S electron microscope.

\section{Results}

We investigated the pineal organ of Ayu and guppy at various stages during the life cycle. The remarkable changes were detected in the outer segment of photoreceptor cell, and the following 8 types were distinguished in the outer segment. These types were termed 1) ciliary, 2) tubular, 3) partially lamellar, 4) wholly lamellar, 5) modifiing lamellar, 6) distorting and swirling lamellar, 7) isolating lamellar, and 8) exhausted types. Fig. 1 shows diagrammatically the features of these types of outer segment. Detailed explanation of these types is as follows. Ciliary type of outer segment was observed at the earliest stage of embryo used in this study. Only ciliary filaments were observed as organelles. The outer segment of tubular type contains irregularly arranged tubules or membranous

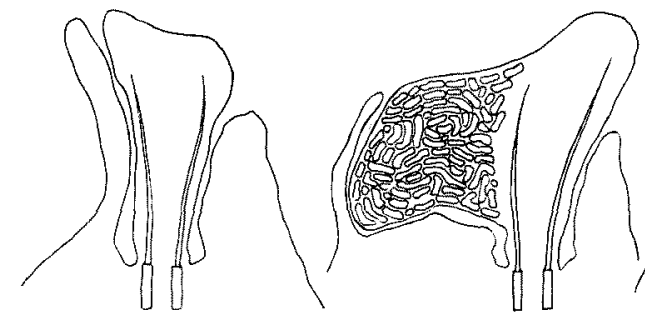

1

2

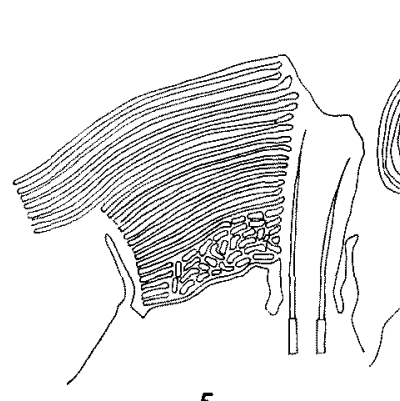

5

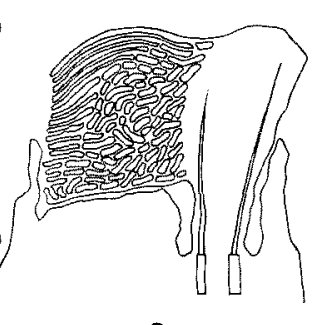

3



4



6

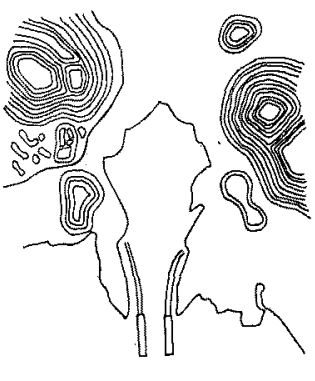

8

Fig. 1. Diagrammatic illustration showing the eight types of outer segments of the photoreceptor cell in fish pineal organ. 1 : ciliary type, 2 : tubular type, $3:$ partially lamellar type, $4:$ wholly lamellar type, 5: modifiing lamellar type, 6: distorting and swriling lamellar type, $7:$ isolating lamellar type, and 8: exhausted type. 
sacs. In the outer segment of partially lamellar type, the lamellar structure arranged perpendicularly to the longitudinal axis of the photoreceptor cell was observed at the localized portion of the outer segment. In the outer segment of wholly lamellar type, regularly arranged lamellae occupy whole portion of the outer segment. This arrangement of lamellae was similar to that in the normal photoreceptor cell of vertebrate retina, though the number of lamellae was fewer in the outer segment of pineal photoreceptor cell. The apical portion in the outer segment of modifiing lamellar type contains a little longer lamellar sacs, in comparison with the basal and central portions of the segment. This indicates the extending modification of the lamellar structure. In the next process of modification, the extended lamellar sacs seem to distort and swirl at the apical portion of the outer segment called distorging and swirling lamellar type. In the final stage of modification, isolated lamellae in distorted and swirled form were detected in the pineal lumen, and residual lamellae and tubules were seen within the outer segment called isolating lamellar type. Lamellae and tubules were not detected in the outer segment of exhausted type.

These types of outer segment described above were seen in the Ayu and guppy at the following stages of life cycle. In the pineal organ of guppy embryo, the outer segments of ciliary, tubular and partially lamellar types were found simultaneously (Figs. 2-4). At this stage, pineal lumen was not detected and the photoreceptor cells were surrounded closely by the supporting cells. To observe the development of the outer segment, developing embryos after artificial fertilization were employed. Most of these embryos hatched on the 17 th day after fertilization. The outer segments of partially and wholly lamellar types were detected in the embryos of 11 and 15 days after fertilization, respectively. The outer segments in the pineal photoreceptor cells of juvenile Ayu were mostly wholly or modifing lamellar types (Figs. 5 and 6). On the other hand, the outer segments of pineal photoreceptor cells in adult guppy were wholly lamellar type in most cases. But even in this type many tubules were observed among the lamellae in the segments. In the adult Ayu, the outer segment of distorting and swirling lamellar and isolating lamellar types were seen often as in the old fish (Figs. 7 and 8). Isolated lamellar materials were also observed abundantly in the pineal lumen. Furthermore, numerous tubules and vesicles were observed in the outer segment of the pineal photoreceptor cells of adult Ayu. The most characteristic type in the outer segment of pineal photoreceptor cell of old Ayu was exhausted one. Lamellae, tubules and other organelles were not detected in the outer segment of this type (Fig. 9). On the other hand, many isolated lamellae were observed in the pineal lumen. The outer segments of wholly lamellar and modifiing lamellar types were seen in the deeper portion of the pineal epithelium. The outer segments of distorting and swirling lamellar and isolating lamellar types were also observed often along the pineal lumen(Figs. 7 and 8). 


\section{Discussion}

Nilsson, ${ }^{1)}$ Yamada and IshiKaWa, ${ }^{2)}$ and WeIdman and KuWABara ${ }^{3)}$ described the developmental process of the retinal photoreceptor cells, using tadpole, human fetus and postnatal rat, respectively. According to these investigators, the outer segment of the photoreceptor cell develops from the ciliary structure at the end of the inner segment. At a somewhat later stage, it is modified to the randomly arranged tubular structures or asymmetrically arranged lamellar sacs. At the final stage, the outer segment of the photoreceptor cell is filled with the stacked lamellar sacs arranged perpendicularly to the longitudinal axis of the cell. Thus, similar structural features to the outer segment of photoreceptor cell seen in adult retina are finished at this stage.

From the present observation using fish embryos, some resemblances were detected between the development of retinal and pineal photoreceptor cells. Namely, the outer segment of pineal photoreceptor cell developed from the outer segment of ciliary type and attained to the outer segment of wholly lamellar type, by way of the outer segment of tubular, and partially lamellar types.

The outer segment of wholly lamellar type in the pineal photoreceptor cell contain about 30 to 70 lamellar sacs, and this is fewer in number than that in visual cell. However, we consider that this number of lamellar sacs is normal in the outer segment of wholly lamellar type in the pineal photoreceptor cell of fish. On the other hand, the synaptic connection between the photoreceptor cell and the nervous elements is already observable in this stage of fish embryo (OMURA and OGURI: unpublished observation). Accordingly, it is suggested that the pineal photoreceptor cell of fish is structurally finished and is functional before hatching.

EAKIN $^{4)}$ reported in lizard, Sceloporus occidentalis, that the vitamin A deficiency brought about remarkable degeneration in the outer segment of photoreceptor cells in lateral and also parietal eyes. Furthermore, KUWABARA and $\operatorname{GoRN}^{5)}$ confirmed in albino rat that the continuous exposure to the visible light of about $750 \mathrm{ft}-\mathrm{c}$ caused the conspicuous damage in retina. In this study they demonstrated that the regular lamellae were transformed into irregular tubules and vesicles at early stage of degeneration. These results indicate that the degeneration of the outer segment of photoreceptor cell is inducible by the various internal and external factors.

In adult $\mathrm{Ayu}$, the outer segment is abundant in tubules and vesicles rather than in lamellae, as shown in the present study and the previous reports. ${ }^{9}{ }^{10}$ ) The adult Ayu inhabits the clear water of shallow river or pond. The diurnal brightness of these circumstances is about 20,000 lux above the water during the summer season. Therefore, the pineal organ seems to be exposed to the strong light through the pineal window. Consequetly, the lamellae of the outer segments might be collapsed and transformed into 
tubules and vesicles in the photoreceptor cells of adult Ayu. However, more detailed supporting experiments are necessary for this explanation.

HoLMGREN $^{11)}$ described in detail the secretory phenomenon in the pineal photoreceptor cells of dogfish, Squalus acanthias. He reported that the outer segment detached from the inner segment was detectable in the central part of the pineal lumen. Dowling and SIDMAN ${ }^{6 / 2}$ recognized in the rat of inherited retinal dystrophy that the swirling sheet or bundles of extracellular lamellae were found among normally developing retinal rods and pigment epithelium. These irregular extracellular lamellae seem to indicate the progressive degeneration of completed outer segment in photoreceptor cell. Autoradiographically, YouNG and BoK $^{7 /}$ did light and electron microscopic observations on frog retina and confirmed that the disc membranes in the outer segment of rod cell moved toward apical portion of the segment and finally were detached from the apical portion. This process of degeneration seems to be endogenously carried out.

In the present observation, the outer segment of modifing lamellar type was found in the pineal photoreceptor cell of juvenile Ayu. Furthermore, the outer segment of distorting and swirling lamellar and isolating lamellar types was detected in the adult Ayu. In addition to these types, the outer segment of exhausted type was found often in the old Ayu, presumably dying soon. In these fishes, isolated lamellar materials were also abundantly observed in the pineal lumen. How to explain such a series of ultrastructural changes observed in the outer segment of pineal photoreceptor cell of fish? We would like to explain this tentatively by the "renewal theory" advocated by HolmGReN, ${ }^{11)}$ and confirmed by YounG. ${ }^{12,13)}$ Namely, probably old and degenerated lamellae are removed from the outer segment and unceasingly new lamellae are formed and supplied. However, further detailed investigations are necessary before such "renewal theory" is adopted for the pineal photoreceptor cells of fish.

We wish to express our cordinal thanks to Prof. T. TAmura, Faculty of Agriculture, Nagoya University, for his valuable advice and encouragment throughout the study. We are also grateful to Dr. K. CHiBA, Fisheries Laboratory of the University of Tokyo, Shizuoka, and Mr. S. IsHII, Gifu Prefectural Fisheries Experiment Station, for the facilities they extended during the collection of fish samples.

\section{References}

1) S. E. G. Nilsson: J. Ultrastr. Res., 11, 581-620 (1964).

2) E. YAMAdA and T. IshikawA: in "The Structure of the Eye" (J, W. Rohen ed.) Vol. 2, 5-16, Schattauer-Verlag, Stuttgart (1965).

3) T. A. Weidman and T. Kuwabara: Arch. Ophthal., 79, 470-484 (1968).

4) R. M. EAKIN: Vision Res., 4, 17-24 (1964).

5) T. Kuwabara and R. A. Gorn: Arch. Ophthal., 79, 69-78 (1968).

6) J. E. Dowling and R. L. Sidman: J. Cell. Biol., 14, 73-109 (1962). 
7) R. W. Young and D. BoK: ibid., 42, 392-403 (1969).

8) Y. Omura and M. Oguri: Bull. Jap. Soc. Sci. Fish., 35, 991-1000 (1969a).

9) Y. Omura, J. KrToH and M. Oguri: ibid., 35, 1067-1071 (1969b).

10) M. Oguri and Y. OMura: in "Responses of Fish to Environmental Changes" (W. Chavin ed.), Charles C. Thomas, Springfield (1971) (in press).

11) H. Holmgren: Arkiv, fur Zool, 11, 1-28 (1918).

12) R. W. Young: J. Cell. Biol., 33, 61-72 (1967).

13) R. W. Young and B. Droz: ibid., 39, 169-184 (1968).

\section{Explanation of the plates}

Figs. 2-4. The outer segments in the embryo of guppy.

Fig. 2: ciliary type, $\times 15,500$, Fig. 3 : tubular type, $\times 25,000$, Fig. 4: partially lamellar type, $\times 18,000$.

Figs. 5 and 6: The outer segments in the juvenile Ayu.

Fig. 5: wholly lamellar type, $\times 21,000$, Fig. 6 : modifing lamellar type, $\times 12,000$.

Figs. 7-9: The outer segments in the old Ayu.

Fig. 7: distorting and swriling lamellar type, $\times 12,000$, Fig. 8 : isolating lamellar type, $\times 10,800$, Fig. 9: exhausted type, $\times 12,000$.

All the specimens shown in these figures were fixed with $1 \% \mathrm{OsO}_{4}$ in Millonig's phosphate buffer exclusive of Fig. 6 fixed with $1.3 \% \mathrm{OsO}_{4}$ in HAMA's collidin buffer. Abbreviations used are as follows. $\mathrm{bb}$ : basal body, $\mathrm{c}$ : centriole, $\mathrm{cc}$ : connecting cilium, il: isolated lamellae, is: inner segment, m: mitochondria, os: outer segment, p: photoreceptor cell, pl: pineal lumen, s: supporting cell. Arrows show terminal bars. 



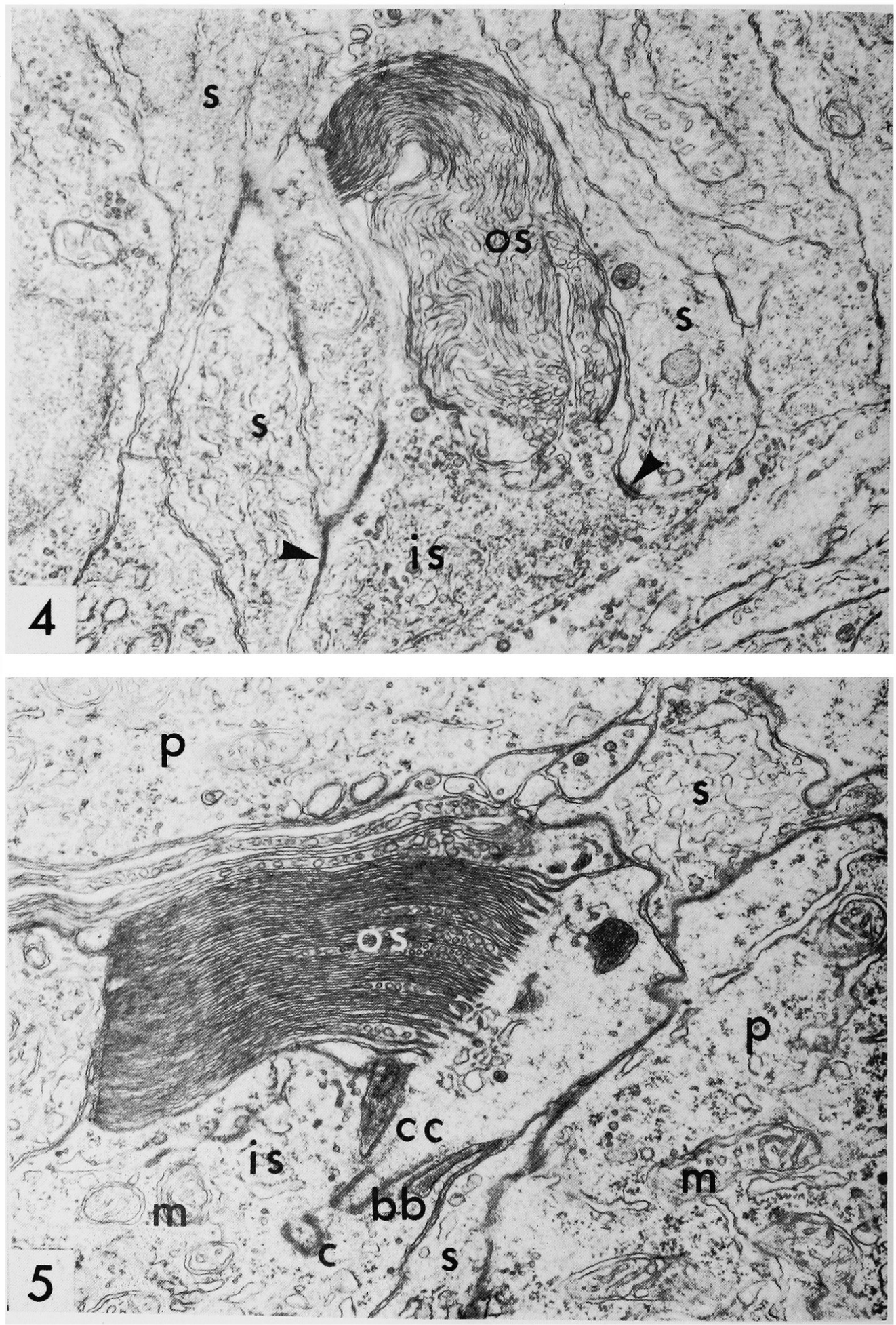

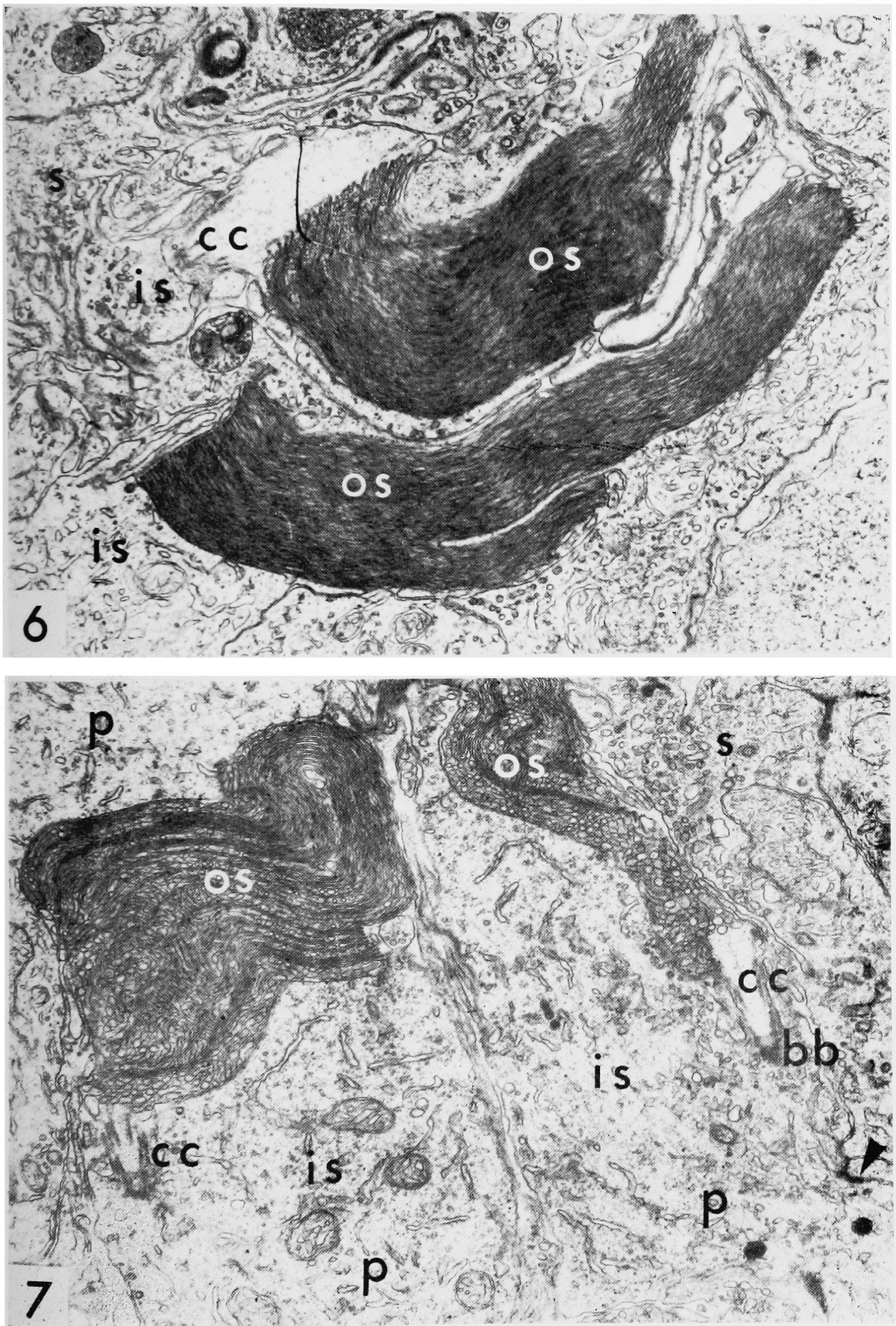

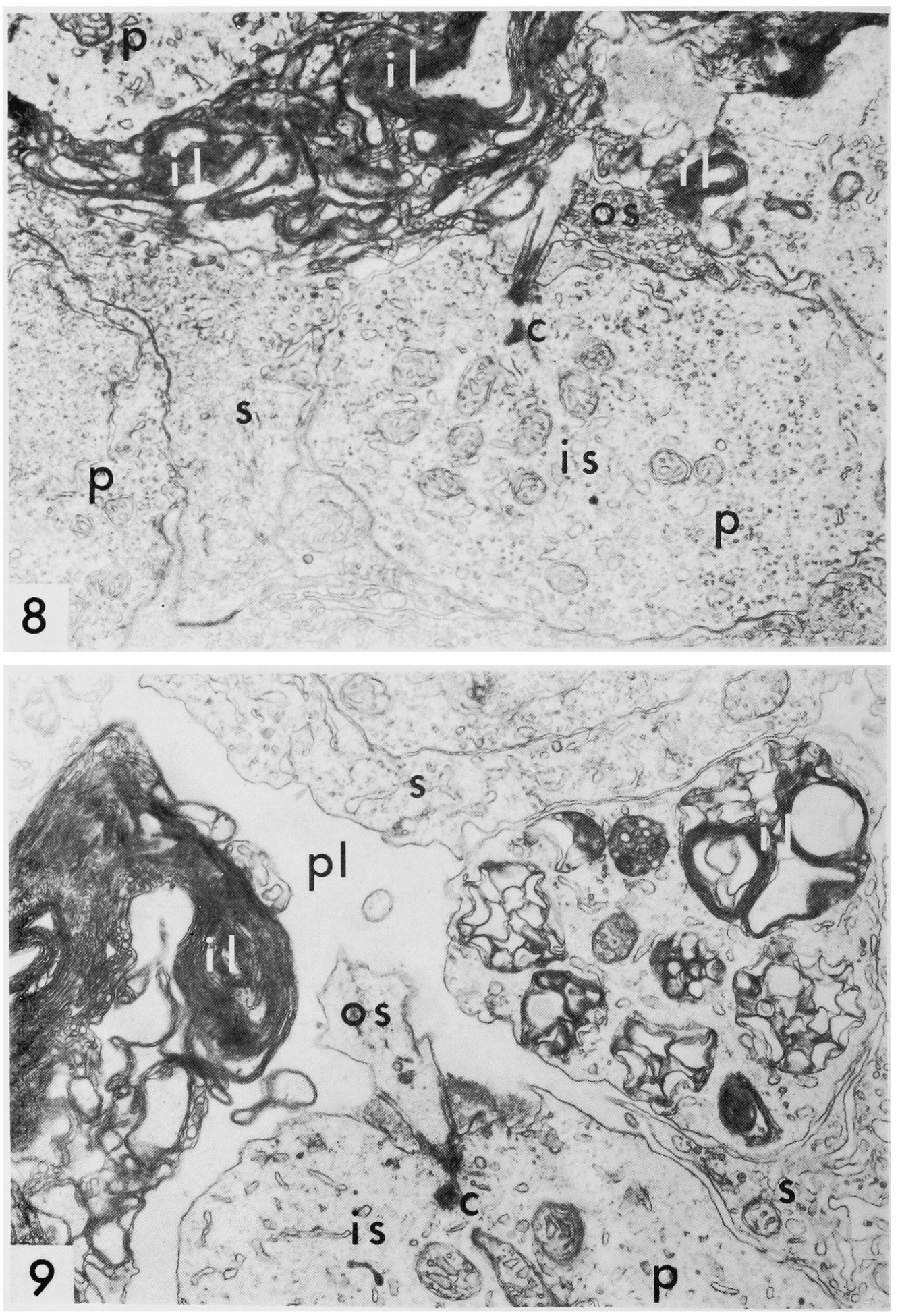